\title{
Large-scale infection of the ascidian Ciona intestinalis by the gregarine Lankesteria ascidiae in an inland culture system
}

\author{
Kaoru Mita ${ }^{1}$, Narudo Kawai ${ }^{1,2}$, Sonja Rueckert ${ }^{1,3}$, Yasunori Sasakura ${ }^{1, *}$ \\ ${ }^{1}$ Shimoda Marine Research Center, University of Tsukuba, Shimoda, Shizuoka 415-0025, Japan \\ ${ }^{2}$ Present address: Department of Biology, Research and Education Center for Natural Sciences, Keio University, \\ 4-1-1 Hiyoshi, Kohoku-ku, Yokohama 223-8521, Japan \\ ${ }^{3}$ Present address: School of Life, Sport \& Social Sciences, Edinburgh Napier University, Sighthill Campus, Sighthill Court, \\ Edinburgh EH11 4BN, UK
}

\begin{abstract}
An important way to keep transgenic and mutant lines of the ascidian Ciona intestinalis, a model system for e.g. genetic functions, in laboratories is via culturing systems. Here we report a disease of $C$. intestinalis observed in an inland culturing system. The disease, called 'long feces syndrome,' is expressed in affected animals by the following characteristic symptoms of the digestive system: (1) excretion of long and thin feces, (2) pale color of the stomach, and (3) congestion of the digestive tube by digested material. Severely diseased animals usually die within a week after the first symptoms occur, implying a high risk of this disease for ascidian culturing systems. The digestive tubes of the diseased animals are occupied by the gregarine apicomplexan parasite Lankesteria ascidiae, suggesting that large-scale infection by this parasite is the cause of long feces syndrome.
\end{abstract}

KEY WORDS: Parasite $\cdot$ Apicomplexan $\cdot$ Digestive tube $\cdot$ Stomach $\cdot$ Congestion $\cdot$ Long feces syndrome $\cdot$ Disease

Resale or republication not permitted without written consent of the publisher

\section{INTRODUCTION}

The ascidian Ciona intestinalis (Linnaeus, 1767) provides an excellent model system to understand the genetic functions in a simplified chordate body plan (Satoh 2003). Rapid embryogenesis, a tadpole larva consisting of a countable number ( 2600) of cells, and quick metamorphosis are advantages for studying the molecular and cellular mechanisms underlying these events. In 2002, the draft genome of this species was determined with about 160 mega base pairs (Mbp) per haploid, encoding 15852 protein-coding genes (Dehal et al. 2002). Basic techniques of molecular biology and embryology for analyzing gene functions have been developed in this ascidian (reviewed by Lemaire 2011). With the help of the genome information and established experi- mental techniques, gene functions of $C$. intestinalis have been extensively studied. Genetic approaches have recently been developed in this ascidian as exemplified by the creation of transgenic lines with transposable elements, enhancer detection, and mutagenesis (Sasakura et al. 2003, 2005, Awazu et al. 2004, Sasakura 2007). These are powerful methods for understanding the functions of genes. The relatively short generation time of this ascidian, approximately 2 to 3 mo for the reproductive stage, helps to carry out genetic approaches. An inland culture system is necessary to conduct $C$. intestinalis genetics, as it allows for the maintenance of genetically modified $C$. intestinalis in laboratories isolated from the ocean. For this reason, it is becoming increasingly important to develop a functioning and maintainable culture system for this ascidian. 
The Shimoda Marine Research Center (SMRC) of the University of Tsukuba (Japan) has established an inland culturing system of Ciona intestinalis similar to that described by Joly et al. (2007). The seawater used for culturing is obtained from the nearby sea at around $3 \mathrm{~m}$ depth without filtration, and the diatom Chaetoceros calcitrans (Paulsen) Takano, 1968 is used as feed. With this system we maintain transgenic, mutant, and inbred lines of $C$. intestinalis in culture.

From late 2010, we frequently observed the excretion of long feces and occasional clogging of the digestive tubes in Ciona intestinalis by digested material. We named this health irregularity 'long feces syndrome' after one of its symptoms. We found that the digestive tubes of diseased animals are occupied by a gregarine apicomplexan, namely Lankesteria ascidiae (Lankester, 1872) Mingazzini, 1891. This species is the type species of the genus Lankesteria (Levine 1977, Rueckert \& Leander 2008) and infects ascidian hosts along with other Lankesteria species.

In this study, we recorded the symptoms of the disease. Lankesteria ascidiae was isolated and documented by means of light microscopy (LM) and scanning electron microscopy (SEM) as well as small subunit (SSU) rDNA sequencing. We conclude that large-scale infection of Ciona intestinalis by the gregarine apicomplexan $L$. ascidiae is the cause of long feces syndrome.

\section{MATERIALS AND METHODS}

\section{Culture conditions}

The inland culture system of Ciona intestinalis is managed according to the suggestions of Joly et al. (2007). Two major adaptations are (1) exclusive use of the diatom Chaetoceros calcitrans as feed, and (2) seawater exchange 3 times a week. The SMRC is equipped with 110 pairs of 10 to $30 \mathrm{l}$ tanks, and currently, over 100 transgenic lines are cultured in total. In the initial step of culturing $C$. intestinalis, we stock about 200 juveniles (about 10 d after fertilization) in a tank. The number of animals is brought down during the successive days according to the growth of animals (on average 30 animals after $3 \mathrm{mo}$ ). Wild or cultured wild-type $C$. intestinalis in this study had been collected from Onagawa Bay (Miyagi), Maizuru Bay (Kyoto), Sagami Bay (Kanagawa), Tosa Bay (Kochi), and Mukaishima (Hiroshima).

\section{Feces addition experiment}

Several hundred fertilized eggs of wild-type Ciona intestinalis and a $\sim 1 \mathrm{~cm}$ long feces string from a diseased animal were added to a $9 \mathrm{~cm}$ petri dish. In total, we added feces strings of 6 diseased animals to petri dishes with fertilized eggs from 4 different clutches. In addition, we had 1 negative control for each clutch with no added feces strings. Two days after $C$. intestinalis completed settlement, the seawater was discarded and new seawater was added to all petri dishes. Seawater was exchanged every other day. Nine days after fertilization, juveniles were stripped off the dishes. After washing once with new seawater, 60 to 100 juveniles for each dish were collected in a $1.5 \mathrm{ml}$ test tube (2 batches from each dish), and their DNA was isolated according to the method described in the following section.

\section{DNA isolation, PCR amplification, cloning, and sequencing}

DNA of Ciona intestinalis juveniles and feces was isolated with a Wizard Genome purification kit (Promega). After precipitation with isopropanol, DNA was dissolved in $20 \mu \mathrm{l}$ water, and $1 \mu \mathrm{l}$ of the DNA solution was subjected to PCR analysis with ExTaq DNA polymerase (Takara Bio). The PCR program was set to 40 cycles of $30 \mathrm{~s}$ at $94^{\circ} \mathrm{C}, 30 \mathrm{~s}$ at $55^{\circ} \mathrm{C}$, and $30 \mathrm{~s}$ at $72^{\circ} \mathrm{C}$, followed by $10 \mathrm{~min}$ at $72^{\circ} \mathrm{C}$ for final extension. The nucleotide sequences of the primers were 5'-ACT GAG AAC TTC CTG CTT GCT C-3' and 5'-GAG CTT GAG AAA TGG CTA CCA C-3'.

DNA from Lankesteria ascidiae was extracted from 50 individual trophozoites that were manually isolated from dissected hosts. They were washed 3 times in filtered and autoclaved seawater, and deposited into a $1.5 \mathrm{ml}$ tube. Genomic DNA was extracted from the cells using the MasterPure complete DNA and RNA purification kit (Epicentre). SSU rDNA sequences were PCR amplified using puReTaq ready-to-go PCR beads (GE Healthcare) and the following eukaryotic PCR primers: F1 5'-GCG CTA CCT GGT TGA TCC TGC C-3' and R1 5'-GAT CCT TCT GCA GGT TCA CCT AC-3' (Leander et al. 2003). The PCR program was set to an initial 4 cycles of $4 \mathrm{~min}, 30 \mathrm{~s}$ at $94^{\circ} \mathrm{C}, 1 \mathrm{~min}$ at $45^{\circ} \mathrm{C}$, and $1 \mathrm{~min} 45 \mathrm{~s}$ at $72^{\circ} \mathrm{C}$, followed by 34 cycles of $30 \mathrm{~s}$ at $94^{\circ} \mathrm{C}, 1 \mathrm{~min}$ at $50^{\circ} \mathrm{C}$, and $1 \mathrm{~min} 45 \mathrm{~s}$ at $72^{\circ} \mathrm{C}$, and then $10 \mathrm{~min}$ at $72^{\circ} \mathrm{C}$ for final extension. PCR products of $L$. ascidiae corresponding to the expected size $(\sim 1800 \mathrm{bp})$ were gel isolated and cloned into the $\mathrm{pSC}$-A-amp/kan vec- 
tor using the StrataClone PCR cloning kit (Agilent Technologies). Nine cloned plasmids, for each PCR product, were digested with ECoRI, and inserts were screened for size using gel electrophoresis. Two identical clones were sequenced with ABI Big-dye reaction mix using vector primers and internal primers oriented in both directions. The GenBank accession number of the SSU rDNA sequence of $L$. ascidiae is JX187607. The SSU rDNA sequences were identified by BLAST analysis, and molecular phylogenetic analyses were performed as described below.

\section{Molecular phylogenetic analyses}

The SSU rDNA sequence from Lankesteria ascidiae was incorporated into an 88-sequence alignment representing the diversity of gregarines, some other important apicomplexan groups, as well as dinoflagellates (outgroup) using MacClade 4 (Maddison \& Maddison 2000) and visual fine-tuning. The program PhyML (Guindon \& Gascuel 2003, Guindon et al. 2005) was used to analyze the 88-sequence alignment (1004 unambiguously aligned positions; gaps excluded) with maximum-likelihood (ML) using a general-time reversible (GTR) model (Posada \& Crandall 1998) incorporating the fraction of invariable sites and a discrete gamma distribution with 8 rate categories $(\mathrm{GTR}+\mathrm{I}+\Gamma+8$ model: $\alpha=0.512$ and $\mathrm{I}=0.092$ for the 88-sequence alignment). ML bootstrap analyses were performed on 100 re-sampled datasets using the same program and the same GTR $+\mathrm{I}+\Gamma+8$ model.

Bayesian analysis of the 88-sequence alignment was performed using the program MrBayes 3.0 (Huelsenbeck \& Ronquist 2001). The program was set to operate with GTR, a gamma-distribution, and 4 Monte Carlo Markov chains (default temperature = 0.2). A total of 2000000 generations were calculated with trees sampled every 50 generations and with a prior burn-in of 100000 generations (2000 sampled trees were discarded; burn-in/convergence was checked manually). A majority rule consensus tree was constructed from 38001 post-burn-in trees. Posterior probabilities correspond to the frequency at which a given node was found in the post-burn-in trees. Independent Bayesian runs on each alignment yielded the same results.

\section{Light and scanning electron microscopy}

Differential interference contrast (DIC) light micrographs of the trophozoites of Lankesteria ascidiae were taken with a system microscope (Olympus BX50) connected to a digital camera (Olympus DP70) or Carl Zeiss AxioImager Z1 connected to a digital camera (Carl Zeiss Axiocam MRm). Individual trophozoites of L. ascidiae $(\mathrm{n}=20)$ were prepared for SEM using the $\mathrm{OsO}_{4}$ vapor protocol described previously (Rueckert \& Leander 2008, 2009). Isolated cells were deposited directly into the threaded hole of a Swinnex filter holder, containing a $5 \mu \mathrm{m}$ polycarbonate membrane filter (Millipore), that was submerged in $10 \mathrm{ml}$ of seawater within a small canister $(2 \mathrm{~cm}$ diameter and $3.5 \mathrm{~cm}$ tall). A piece of Whatman filter paper was mounted on the inside base of a beaker $(4 \mathrm{~cm}$ diameter and $5 \mathrm{~cm}$ tall) that was slightly larger than the canister. The Whatman filter paper was saturated with $4 \% \mathrm{OsO}_{4}$, and the beaker was turned over the canister. The parasites were fixed by $\mathrm{OsO}_{4}$ vapors for $30 \mathrm{~min}$. Ten drops of $4 \% \mathrm{OsO}_{4}$ were added directly to the seawater, and the parasites were fixed for an additional $30 \mathrm{~min}$ on ice. A $10 \mathrm{ml}$ syringe filled with distilled water was screwed to the Swinnex filter holder, and the entire apparatus was removed from the canister containing seawater and fixative. The parasites were washed and then dehydrated with a graded series of ethyl alcohol. Prepared specimens were freeze dried with t-butanol. Filters were mounted on stubs, sputter coated with $5 \mathrm{~nm}$ gold, and viewed under SEM (JEOL NeoScope JCM5000).

\section{RESULTS}

\section{Symptoms of long feces syndrome of Ciona intestinalis}

Long feces syndrome shows 3 typical symptoms. First, feces of the diseased animals become longer and thinner than those of healthy animals (Fig. 1A,B). Sometimes diseased animals eject white feces instead of brown. The brown color is derived from the color of the fed diatoms. Second, the digested material is clogged in the stomach or intestine (Fig. 1C). In this case, the animals do not stop feeding for a while, and therefore the amount of congested digested material continues to increase. The clogged material has a glomerular shape of long feces (Fig. 1D). The third symptom refers to the color of the stomach. Stomachs of healthy animals are of a distinct orange color (Fig. 1A). In contrast, the stomachs of diseased animals become pale (Fig. 1B). These 3 symptoms do not always occur at the same time (Fig. 1C). Usually, the first symptom we recognize in 

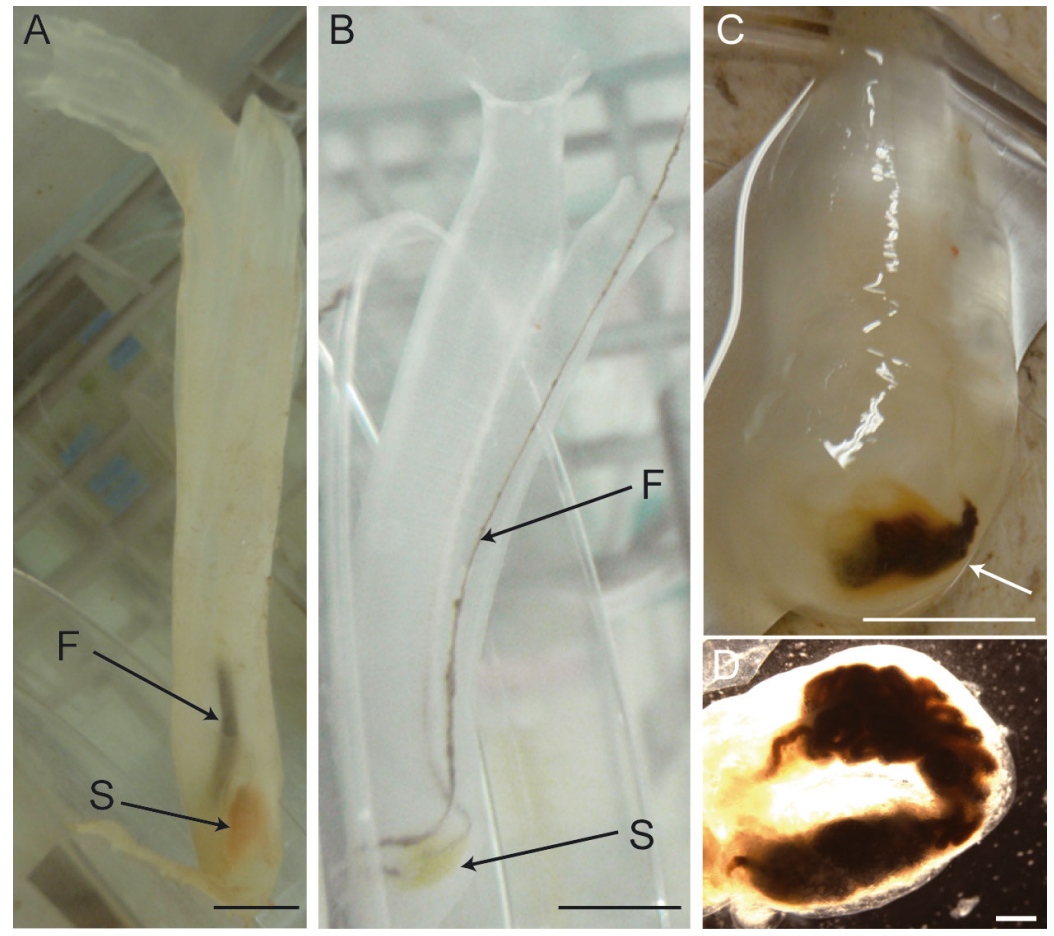

tive to the growth stage also differs among animals. We frequently noticed the long feces syndrome in $\sim 3 \mathrm{~cm}$ long (about 1.5 mo old) individuals, although smaller animals about $5 \mathrm{~mm}$ long or older animals cultured for several months sometimes started to show the symptoms.

\section{Gregarine apicomplexans in the digestive tube of diseased animals}

All symptoms of the long feces syndrome are related to the digestive tube. We observed the digestive tube of diseased animals and found that many gregarines, namely Lankesteria ascidiae, were present in the tube (Fig. 2A-C). Although some gregarines could be found in healthy animals, they were dispersed throughout the digestive tubes (Fig. 2A). In conFig. 1. Ciona intestinalis. Long feces syndrome. (A) Healthy adult. The stomach (S) is orange. F: feces in the intestine. (B) Adult showing long feces syndrome. Its stomach is pale in color. The animal excreted feces that are longer than its body length. Note that the feces are thinner than in (A). (C) Adult showing clogging of the digestive tube with digested materials (arrow). The stomach of this animal was orange. (D) Congested digested materials in the intestine are glomerulous long feces. Scale bars $=(A-C) 10 \mathrm{~mm}$, (D) $1 \mathrm{~mm}$

trast, the stomach and intestine of the diseased animals were completely occupied by the gregarines (Fig. 2B,C). The stomachs of severely infected animals were sometimes swollen due to the number of gregarines (Fig. 2B). When we examined the stomachs of about $5 \mathrm{~mm}$ long, 1 mo old animals,

diseased animals is the long feces, and then digested material is clogged in the digestive tubes of some animals, and the color of the stomachs becomes paler. The tunics of the diseased animals, the thin mantles surrounding their bodies, shrink during the progression of the disease. Soon after, they stop feeding entirely and die. The time from the onset of the disease to death varies widely among animals and is therefore difficult to determine. When the symptoms progress rapidly, the diseased animals come to the serious stage of the disease and death within $1 \mathrm{wk}$ after recognition.

All transgenic, mutant, and inbred lines in our culturing system suffered from the disease, suggesting that a specific genetic modification or genetic background is not the cause of the disease. Our rough estimation was that $52 \%$ of the tanks $(n=25)$ had at least 1 diseased animal, and approximately $7 \%$ of animals in total $(\mathrm{n}=678)$ showed the syndrome; however, the numbers are underestimated because we eliminate diseased animals as soon as possible to avoid further infection. The onset of the disease rela- both large and small gregarines were observed (Fig. 2D), suggesting that the infection with gregarines starts as early as that stage.

\section{Morphology of Lankesteria ascidiae}

The trophozoites of Lankesteria ascidiae showed a variety of different cell shapes (Fig. 3A-E). Usually the cell had a broad anterior end that narrowed into a blunt posterior end (Fig. 3A,C,E). Some of the cells were more oval, spindle shaped (Fig. 3B) or oblong to pin shaped (Fig. 3D). Trophozoites were brownish in color, suggesting an accumulation of amylopectin granules within the cytoplasm. The nucleus was located in the anterior half of the cell for most trophozoites (Fig. 3A,C,D). The nucleus of some cells was located in the cell center (Fig. 3B). The mucron area was round to pointed and free of amylopectin granules (Fig. 3A-D). The trophozoites varied in size. Trophozoites were $62 \mu \mathrm{m}(33-92 \mu \mathrm{m}, \mathrm{n}=33)$ long and $19 \mu \mathrm{m}(9-36 \mu \mathrm{m}, \mathrm{n}=33)$ wide, measured at the 

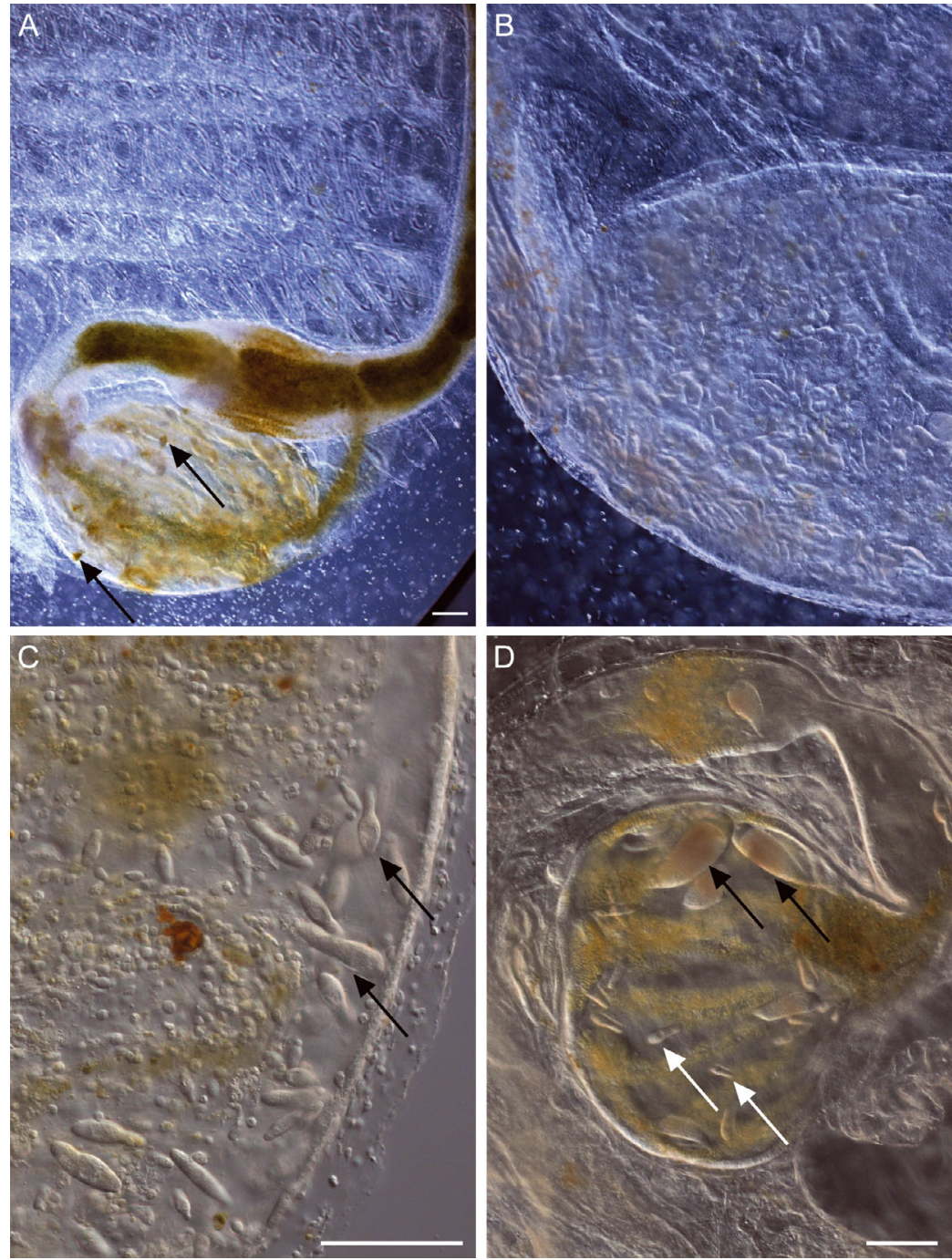

Fig. 2. Ciona intestinalis. (A) Stomach and intestine of a healthy animal. Some gregarines are observed (2 examples are shown by arrows). Stomach folds can be recognized as light brown stripes. (B) Stomach and intestine of a diseased animal. The ducts are filled with gregarines. No folds are recognizable. (C) High-magnification image of gregarines (2 examples are shown by arrows) in the intestine. (D) Gregarines in the stomach of a young ( $5 \mathrm{~mm}$ long) animal. Black and white arrows indicate examples of extraordinarily large and small gregarines, respectively. Scale bars $=100 \mu \mathrm{m}$

widest part of the cell. The spherical nucleus was $9 \mu \mathrm{m}(6-13 \mu \mathrm{m}, \mathrm{n}=30)$ in diameter. The trophozoites were relatively stiff and capable of gliding motility (see Movie 1 in the supplement at www.int-res.com/ articles/suppl/d101p185_supp/). Some trophozoites were capable of slight bending movements. Trophozoites possessed dense arrays of epicytic knobs across the entire surface of the cell (Fig. 3E,F), including the mucron. The knobs were about 0.11 to $0.13 \mu \mathrm{m}$ in diameter (Fig. 3F). The density of epicytic knobs was between 120 and 144 knobs $\mu^{-1}$ (Fig. 3F).
Epicytic folds were almost entirely absent. If present, the longitudinal folds were not continuous across the length of the cell. More epicytic folds were present on the posterior tail-like region of the trophozoites than on the anterior broader part (Fig. 3E).

\section{Molecular phylogeny of Lankesteria ascidiae}

The isolated gregarines were classified as $L$. ascidiae within the genus Lankesteria based on their morphological characteristics and host species (following Ormières 1965, Levine 1981, Ciancio et al. 2001). However, there were no pre-existing molecular sequence data available for this species. As $L$. ascidiae is the type species of the genus Lankesteria, we examined the phylogenetic position of $L$. ascidiae by molecular phylogenetic analysis. We isolated and sequenced the SSU rDNA of $L$. ascidiae, and a phylogenetic tree was constructed with the SSU rDNAs of other gregarines and gregarinerelated species retrieved from GenBank. The 88-taxon dataset recovered a moderately supported clade of apicomplexans, albeit with a poorly resolved backbone (Fig. 4). Within the apicomplexans, 3 clades were formed, consisting of (1) piroplasmids, coccidians, and rhytidocystids; (2) cryptosporidians; and (3) neogregarines, monocystids, archigregarines, and eugregarines. The new sequence from $L$. ascidiae clustered within a strongly supported clade of mainly marine eugregarines including urosporids (i.e. Pterospora), lecudinids (Lecudina, Lankesteria, Difficilina), and archigregarines (i.e. Veloxidium). The genera Lecudina, Lankesteria, and several environmental sequences formed a highly supported clade within the marine eugregarines (Fig. 4). This Lankesteria-Lecudina clade consisted of 3 main subclades: (1) Lankesteria spp., (2) Lecudina tuzetae and an environmental sequence (AY179977), and (3) Lecudina longissima and Lecudina phyllochaetopteri. Within the Lankesteria subclade, $L$. ascidiae, L. chelyosomae and $L$. cystodytae formed a sister clade to $L$. abbotti. 


\section{Spreading of gregarines to new hosts mediated by feces}

In order to understand the mechanisms of gregarine infection here, we developed a sensitive method to detect gregarines at an early stage of infection by using the SSU rDNA isolated above. A pair of oligonucleotide primers specific to the DNA was designed, and PCR-based detection of gregarines was conducted. It is generally accepted that gregarines are released with host feces. PCR analysis of the feces of the diseased animals indicated that the long feces contained gregarines (Fig. 5A; 100\%, $\mathrm{n}=10$ ). After careful observation of the feces under the microscope, we found trophozoites of gregarines in the brown long feces, but in very low numbers. In contrast, the white feces were composed almost entirely of gregarines (Fig. 5B). In the feces, we mostly found small and round oocysts $(\sim 7 \mu \mathrm{m}$ in diameter). We also found cysts of $\sim 50 \mu \mathrm{m}$ diameter in both white and brown feces (Fig. 5C). Our PCR analysis suggests that these cysts are the gametocysts of the gregarines (Fig. 5D).

In order to prove that Ciona intestinalis are infected with gregarines through the feces of diseased animals, we performed an experiment in which we added feces of diseased animals to fertilized eggs. After $1 \mathrm{wk}$ of culturing under the same conditions as the transgenic lines, DNA isolated from the whole body of the juveniles was subjected to PCR analysis in order to detect gregarines. DNA from the groups to which the feces were added showed the PCR band corresponding to the gregarine (Fig. 5E). Four out of 6 feces caused gregarine infection, while negative controls without feces did not show any infection. Although we carefully observed these juveniles for gregarines, none was recognized in their bodies.

During normal culturing procedures, sperm of transgenic animals and wild-type eggs are surgically collected and then mixed for insemination. Throughout collection of germ cells, contamination with the feces may occur, because the gonoducts are located
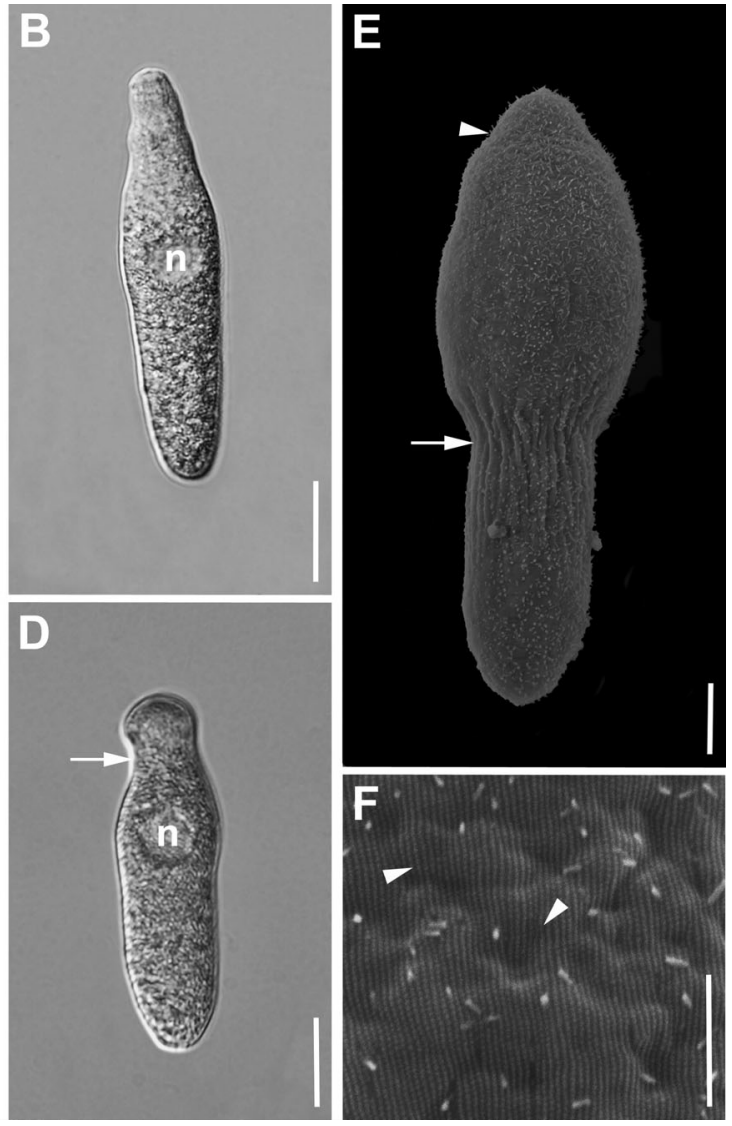

Fig. 3. Lankesteria ascidiae. Differential interference contrast and scanning electron micrographs (SEM) of gregarines isolated from the cultured ascidian Ciona intestinalis. (A-D) Individual trophozoites showing morphological differences. Most cells had a broadened anterior end that narrowed into a blunt posterior end. Some of the cells were more oval, spindle shaped or oblong to -shaped. Trophozoites were brownish, with granular cytoplasm. The nucleus (n) was located in the anterior half of the cell for most trophozoites or in the cell middle of some. The mucron area was round to pointed and free of the cell. (E) SEM showing the general morphology and surface ultrastructure . ascidiae. The mucron (arrowhead) can be seen as slight protrusion from the cell body proper. The trophozoites did not have longitudinal epicytic folds inscribing the surface of the whole cell. They only had a few folds on parts of the body, mostly in the posterior half (arrow). (F) High-magnification SEM of the surface ultrastructure revealing rows of small surface knobs (arrowheads). Scale bars $=(A-D) 20 \mu \mathrm{m},(E) 5 \mu \mathrm{m},(\mathrm{F}) 2 \mu \mathrm{m}$

Fig. 4. Maximum-likelihood tree of apicomplexans and dinoflagellates (outgroup) as inferred using the GTR model of nucleotide substitutions, a gamma distribution, and invariable sites on an alignment of 88 SSU rDNA sequences and 994 unambiguously aligned sites $(-\ln \mathrm{L}=17934.96, \alpha=$ 0.521 , fraction of invariable sites $=0.092,4$ rate categories). Numbers at the branches denote maximum likelihood (ML) bootstrap percentages (top) and Bayesian posterior probabilities (bottom). Black dots on branches denote Bayesian posterior probabilities of 0.95 or higher and ML bootstrap percentages of $95 \%$ or higher. The sequence addressed in this study is highlighted by the black box 


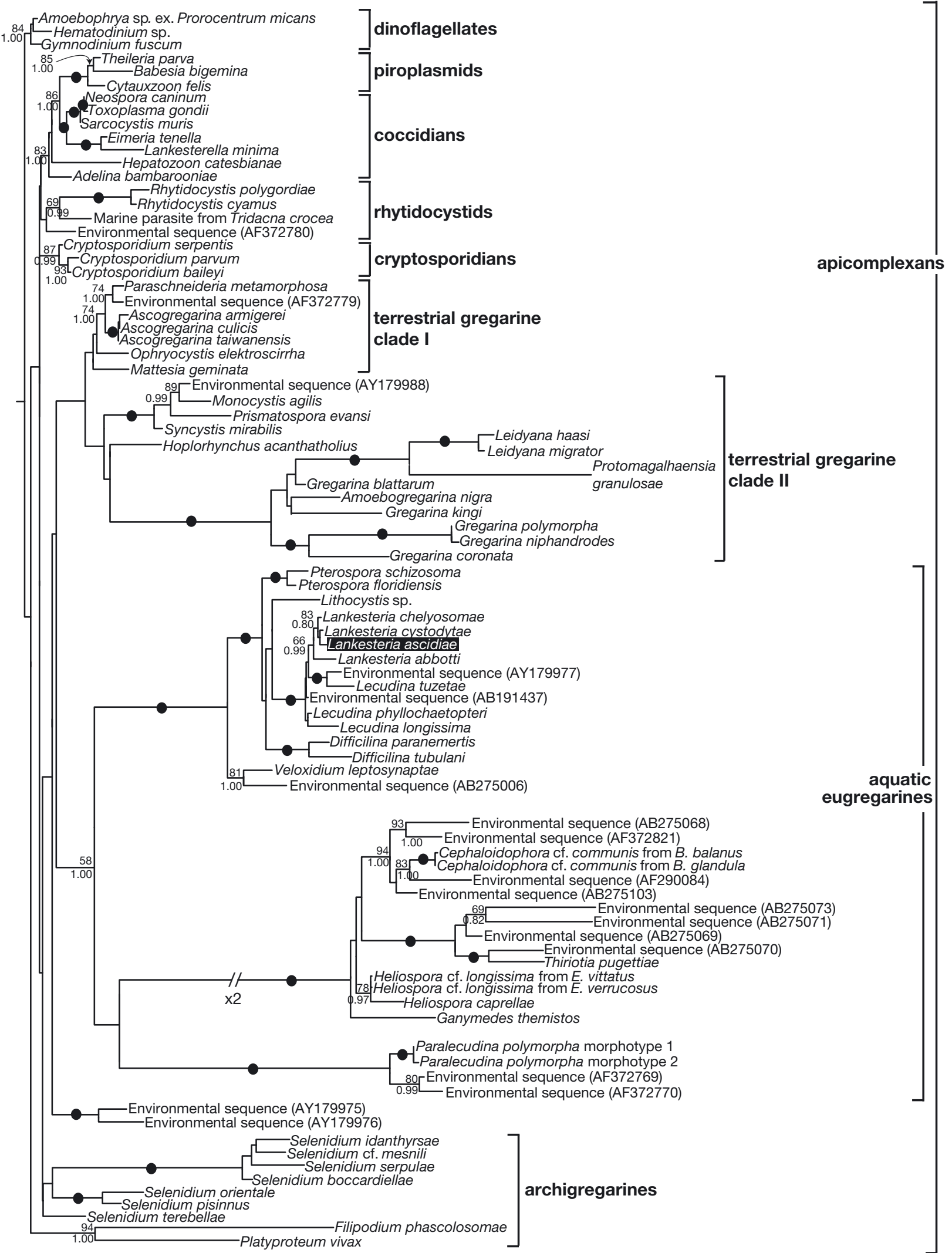

Est. 0.2 substitutions/site 
A
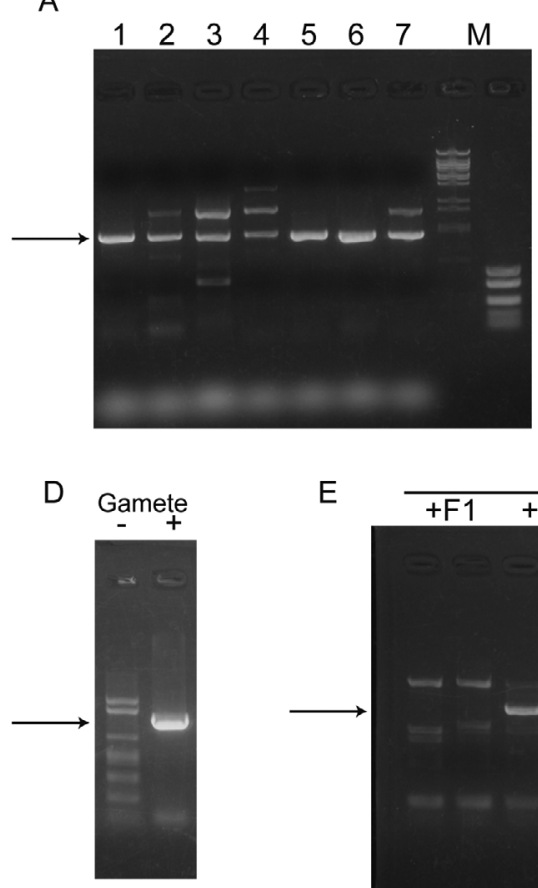

$E$
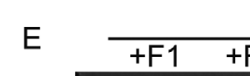

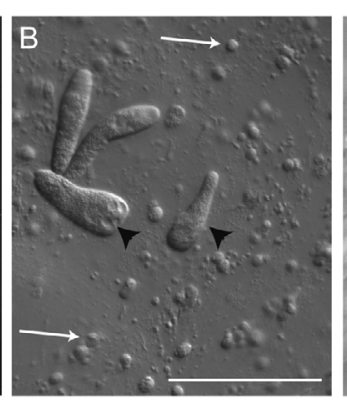

Clutch 1

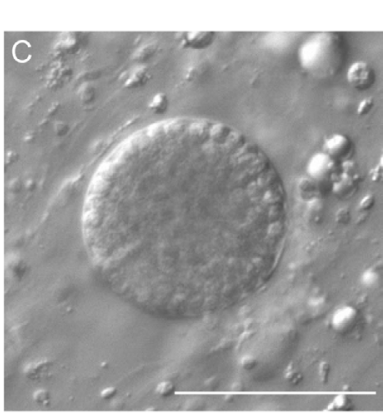

Clutch 2
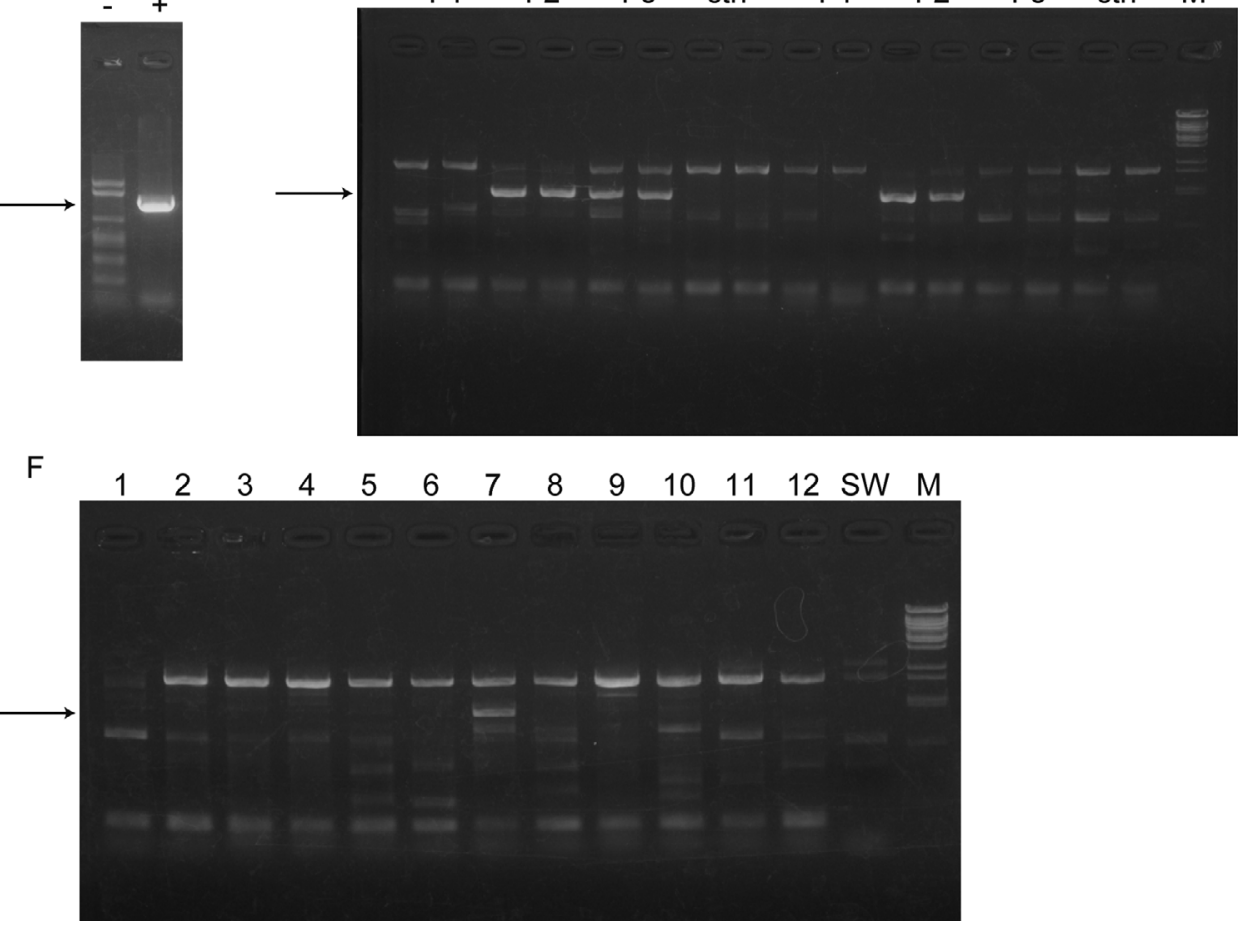

Fig. 5. Ciona intestinalis infected by Lankesteria ascidiae. (A) PCR analysis of long feces of diseased animals detected the presence of gregarines in the feces. 1-7: PCR products from the DNA isolated from the feces of 7 diseased animals. The arrow indicates the appropriate size for the DNA of the gregarine. M: marker lane. (B) Gregarines found in the feces of a diseased animal. White arrows indicate 2 examples of round oocysts; black arrowheads indicate trophozoites. (C) Gametocyst of the gregarine observed in feces. (D) DNA from feces without (-) and with (+) gametocysts were analyzed by PCR. DNA with gametocysts showed bands corresponding to gregarines (arrow). (E) Juveniles cultured with feces of diseased animals are infected by gregarines. Shown are the results of 2 clutches of eggs with added feces from 3 diseased animals (F1-F3). For each combination of clutches and feces, DNA from 2 batches of juveniles was analyzed. In Clutch 1, Feces 2 and 3 caused infection, and in Clutch 2, Feces 2 caused infection. ctrl: negative controls of juveniles cultured without feces. Arrow indicates the position of the PCR bands suggesting gregarine infection. (F) Juveniles isolated by the normal procedure of culturing are infected by gregarines. Of 12 families subjected to PCR analysis, 1 (lane 7) showed the band specific to the gregarine (arrow). SW: negative control for PCR of the seawater sample without juveniles as a template. Scale bars = (B) $100 \mu \mathrm{m}$, (C) $50 \mu \mathrm{m}$ very close to the intestine. To examine whether juveniles grown under the normal culturing conditions were infected with gregarines, juveniles of Ciona intestinalis at $\sim 10 \mathrm{~d}$ after fertilization were subjected to PCR analysis. We examined juveniles of 12 clutches, and found that one of them tested positive for the gregarine (Fig. 5F).

\section{DISCUSSION}

\section{Long feces syndrome}

In the present study, we describe the large-scale infection of cultured Ciona intestinalis by the gregarine Lankesteria ascidiae. Severely infected animals showed characteristic symptoms. The descrip- tion of the symptoms, viz. excretion of long and thin feces, pale color of the stomach, and congestion of the digestive tube by digested material, facilitates a quick recognition of the infection. Being able to identify a large-scale infection by its symptoms means there is no need for observing the digestive tube of animals, which will kill them. The long feces of the diseased animals are probably caused by the large amount of gregarines present in their digestive tubes. The stomach of $C$. intestinalis has many folds (Millar 1953), and food probably passes through the folds for efficient digestion. The presence of many gregarines in the stomach may decrease the efficiency of digestion by either reducing physical contact to the epithelial layer of the stomach, or by absorbing nutrients from the stomach. According to Siedlecki (1901), L. ascidiae has 2 
different feeding habits. The infective sporozoites penetrate and destroy gastric epithelial cells and even deeper tissues, whereas the trophozoites remain free in the gastric lumen or attached to epithelial cells, while absorbing nutrients via the cell surface. The digested materials of the diseased animals are sticky and they maintain the thin tubular structure after they are transferred to the intestine. Gregarines are known to produce mucus (Vávra \& Small 1969), which might make the feces sticky. The intestines of diseased animals are also occupied by many gregarines, restricting the space of the digestive tube for excretion, which is another cause of long feces and the congestion of the digestive tube with digested material. Diseased animals often exhibit a pale stomach color compared to the bright orange stomach of healthy animals. There are 2 possible explanations for this. One is that the reduced nutrient absorbance by the diseased animals causes a loss in the productivity of the orange substance, and the other is that the gregarines might absorb the orange substance from the stomach. Gregarines are also present in healthy animals, suggesting that a gregarine infection does not always cause strong negative effects on the health of $C$. intestinalis. The degree of infection in our culturing system apparently exceeds the limit for $C$. intestinalis to cope with the infection. The seriously infected animals probably die for 2 reasons: insufficient nutrient uptake and physical difficulties in excreting digested materials.

\section{Lankesteria ascidiae}

Lankesteria ascidiae belongs to the group of eugregarines that infect ascidian hosts and is the type species of the genus Lankesteria (Levine 1977, Rueckert \& Leander 2008). Some reports indicate that Lankesteria species are quite host specific (Levine 1981, Rueckert \& Leander 2008), while others have observed the same species in different hosts (Leander et al. 2006). The genus once contained gregarines infecting several different phyla but is now referred to as exclusive for ascidians (Levine 1977, Ormières 1965, Rueckert \& Leander 2008). Gregarine apicomplexans are unicellular parasites that can infect the intestine, coelom, and reproductive vesicles of invertebrates (Leander 2008). The impact of gregarine apicomplexan parasites on their hosts varies from harmless to severe: commensalism, reduction of a host's ability to assimilate food, delayed development, and decreased body weight and longevity
(Zuk 1987, Lightner 1993, Åbro 1996, Siva-Jothy \& Plaistow 1999, Jiménez et al. 2002, Prokopowicz et al. 2010).

Eugregarines usually possess longitudinal epicytic folds that cover and thus enlarge the surface and provide surface-mediated nutrition. In some Lankesteria species such as L. parascidae, L. morchellii, L. chelyosomae, and L. cystodytae (Ormières 1972, Rueckert $\&$ Leander 2008), the folds are absent or very reduced and instead there are surface knobs (syn. tubercles). Ciancio et al. (2001) also described the surface knobs for $L$. ascidiae by means of transmission electron microscopy. We were able to show the surface knobs of $L$. ascidiae by SEM revealing a dense array of the knobs. Epicytic folds are also known to play an important role in gliding motility (Vávra \& Small 1969, Heintzelman 2004). As already seen in $L$. chelyosomae and L. cystodytae (Rueckert \& Leander 2008), L. ascidiae is capable of gliding motility (Movie 1 in the supplement), even though all these lack a continuous coverage of epicytic folds.

The SSU rDNA sequence of Lankesteria ascidiae clusters as expected within the clade of other aquatic eugregarines, more specifically within a small clade consisting of other Lankesteria species. Within this clade, there is 1 species that possesses surface folds (L. abbotti) and 3 species that possess surface knobs (L. chelyosomae, L. cystodytae, and L. ascidiae). As more sequences of ascidian-infecting gregarines become available it will become more clear whether the surface structure of the Lankesteria species is reflected in the phylogenetic relationships within this genus.

\section{Transmission pathways}

In the usual route of gregarine infection, a new host organism must ingest gregarine cysts to become infected. After ingestion, sporozoites hatch from the oocysts and penetrate the host cells. The sporozoites enlarge and become trophozoites, emerge from the cell, and start feeding. Trophozoites within a host pair up in a process called 'syzygy' and develop into gamonts. The gamontocyst forms around the pair of gamonts. Each gamont divides into numerous gametes. A male and female gamete fuse to form a zygote that is then surrounded by an oocyst wall. Within the oocyst, meiosis occurs to yield 4 (or more) spindle-shaped sporozoites (Kuriyama et al. 2005). Hundreds of oocysts accumulate within each gametocyst (cf. Levine 1977). The cysts are usually released via host feces or via host death, and they can 
remain in the environment for an extended period of time until a new host ingests them.

Our study shows that feces of diseased Ciona intestinalis are the major mediator of the gregarine infection. In our closed system, the next host organism is readily available for the gregarines. Adding feces to the fertilized eggs in our experiment caused the gregarine infection, suggesting that contamination of the eggs and sperm with feces during progeny isolation is another cause of spreading the infection. We were surprised that animals subjected to gregarine-containing feces in their early developmental stage (during the first $2 \mathrm{~d}$ after fertilization) were infected by the gregarines, although animals at this stage only have a premature mouth and hence do not feed. For this reason, the gregarines cannot enter the body of $C$. intestinalis through the feeding action of the host. This might hint at an unusual route of gregarine infection. Because the seawater was exchanged before the juveniles started feeding, all gregarines and cysts floating in the seawater or loose on the bottom of the petri dish should have been removed. Therefore, Lankesteria ascidiae must have a way to resist the seawater exchange, perhaps by some form of attachment to the hosts or petri dishes. The stickiness of the excreted host feces might play a role in this.

Avoiding feces contamination during progeny isolation is essential to minimize the risk of infection among new generations. PCR testing is an easy and reliable way to detect progeny families infected with the gregarines. In addition, removing diseased animals as early as possible is also an effective way to avoid expansion of the infection. We have experienced that the frequent exchange of seawater and reducing the amount of feed sometimes improves the symptoms of the disease. This way, feces with excreted gregarines are removed soon, which should decrease the number of gregarines in the infected ascidians over time. Feed reduction will avoid congestion of digested materials.

A line of evidence suggests that feces are not the only source of gregarine contamination in our culturing system. We have been culturing inbred lines of Ciona intestinalis for over $3 \mathrm{yr}$. These animals do not have any contact with animals from other populations. However, they have suffered from large-scale infections of gregarines since 2010. Inheritance of gregarines in the inbred lines from their founder animals is not realistic. In addition, gregarines started to cause severe negative effects in recent ascidian generations at the same time as problems with the transgenic lines occurred. We therefore favor the idea that the origin of gregarine infection must come from a source that is used for culturing both transgenic and inbred lines. In our culture system, the components that are used in both lines are the diatom feed and the seawater. The diatoms as a possible source for the gregarine infection can be neglected, because we use a pure-cultured commercial product. Hence, the gregarines could only enter the system through the seawater that is obtained in the nearby ocean. At present, we collect seawater at $3 \mathrm{~m}$ depth without filtration. The ocean at the point of collection is $4 \mathrm{~m}$ deep with a rocky seafloor surrounded by a sandy area. It is possible that floating cysts of the gregarines are collected with the seawater when the seawater is turbulent due to heavy wave action. Sterilization of the collected seawater by heat or filtration might be a solution to avoid future gregarine infections.

The present study describes the long feces syndrome of Ciona intestinalis in a culture system, its causal gregarine infection, and plausible countermeasures for the syndrome. It is important to share the knowledge about the risk of this syndrome amongst the $C$. intestinalis culturing community to be able to improve the inland culture system of this ascidian. Ironically, the large-scale infection of $C$. intestinalis by the gregarine Lankesteria ascidiae in our culture system provides us with an excellent experimental model to study the mechanisms of infection and development of this gregarine species.

Acknowledgements. We thank members of the SMRC (University of Tsukuba) for their kind cooperation with our study, and S. Fujiwara, K. Tagawa, N. Yamaguchi, and all members of the Maizuru Fishery Research Station of Kyoto University, Misaki Marine Biological Station, The University of Tokyo, the Education and Research Center of Marine Bioresources at Tohoku University, and the Marine Biological Laboratory, Graduate School of Science at Hiroshima University for the collection of Ciona adults. We are grateful to A. Kumagai for helpful discussion. This study was supported by Grants-in-Aid for Scientific Research from the Japan Society for the Promotion of Science and the Ministry of Education, Sports, Science and Technology to Y.S. This study was further supported by grants from the National BioResource Project.

\section{LITERATURE CITED}

Åbro A (1996) Gregarine infestation of adult Calopteryx virgo L. (Odonata: Zygoptera). J Nat Hist 30:855-859

Awazu S, Sasaki A, Matsuoka T, Satoh N, Sasakura Y (2004) An enhancer trap in the ascidian Ciona intestinalis identifies enhancers of its Musashi orthologous gene. Dev Biol 275:459-472

Ciancio A, Scippa S, Cammarano M (2001) Ultrastructure of trophozoites of the gregarine Lankesteria ascidiae (Api- 
complexa: Eugregarinida) parasitic in the ascidian Ciona intestinalis (Protochordata). Eur J Protistol 37:327-336

Dehal P, Satou Y, Campbell RK, Chapman J and others (2002) The draft genome of Ciona intestinalis: insight into chordate and vertebrate origins. Science 298: 2157-2167

Guindon S, Gascuel O (2003) A simple, fast, and accurate algorithm to estimate large phylogenies by maximum likelihood. Syst Biol 52:696-704

Guindon S, Lethiec F, Duroux P, Gascuel O (2005) PHYML online - a web server for fast maximum likelihoodbased phylogenetic inference. Nucleic Acids Res 33: W557-W559

Heintzelman MB (2004) Actin and myosin in Gregarina polymorpha. Cell Motil Cytoskeleton 58:83-95

Huelsenbeck JP, Ronquist F (2001) MrBayes: Bayesian inference of phylogenetic trees. Bioinformatics 17:754-755

> Jiménez R, de Barniol L, Machuca M (2002) Nematopsis marinus n. sp., a new septate gregarine from cultured penaeoid shrimp Litopenaeus vannamei (Boone), in Ecuador. Aquacult Res 33:231-240

> Joly JS, Kano S, Matsuoka T, Auger H and others (2007) Culture of Ciona intestinalis in closed systems. Dev Dyn 236:1832-1840

Kuriyama R, Besse C, Gèze M, Omoto CK, Schrével J (2005) Dynamic organization of microtubules and microtubuleorganizing centers during the sexual phase of a parasitic protozoan, Lecudina tuzetae (Gregarine, Apicomplexa). Cell Motil Cytoskeleton 62:195-209

Leander BS (2008) Marine gregarines: evolutionary prelude to the apicomplexan radiation? Trends Parasitol 24:60-67

Leander BS, Clopton RE, Keeling PJ (2003) Phylogeny of gregarines (Apicomplexa) as inferred from small-subunit rDNA and beta-tubulin. Int J Syst Evol Microbiol 53: 345-354

Leander BS, Lloyd SAJ, Marshall W, Landers SC (2006) Phylogeny of marine gregarines (Apicomplexa)-Pterospora, Lithocystis and Lankesteria - and the origin(s) of coelomic parasitism. Protist 157:45-60

Lemaire P (2011) Evolutionary crossroads in developmental biology: the tunicates. Development 138:2143-2152

Levine ND (1977) Revision and checklist of the species (other than Lecudina) of the aseptate gregarine family Lecudinidae. J Protozool 24:41-52

Levine ND (1981) New species of Lankesteria (Apicomplexa, Eugregarinida) from ascidians on the Central California Coast. J Protozool 28:363-370

Lightner DV (1993) Diseases of cultured penaeid shrimp. In: McVey JP (ed) Handbook of mariculture, 2nd edn, Vol 1. CRC Press, Boca Raton, FL, p 393-486

Maddison DR, Maddison WP (2000) MacClade 4. Sinauer Associates, Sunderland, MA

Millar RH (1953) Ciona. LM.B.C. Memoirs XXXV. Liverpool University Press, Liverpool

Editorial responsibility: Stephen Feist, Weymouth, UK
Ormières R (1965) Recherches sur les sporozoaires parasites de tuniciers. Vie Milieu 15:823-946

Ormières R (1972) Études ultrastructurale des Lankesteria, eugrégarines parasites de tunicieres. Comparison avec les 'Lankesteria' parasites des diptères. C R Hebd Seances Acad Sci 274:3254-3257

Posada D, Crandall KA (1998) MODELTEST: testing the model of DNA substitution. Bioinformatics 14:817-818

> Prokopowicz AJ, Rueckert S, Leander BS, Michaud J, Fortier L (2010) Parasitic infection of the hyperiid amphipod Themisto libellula in the Canadian Beaufort Sea (Arctic Ocean), with a description of Ganymedes themistos sp. n. (Apicomplexa, Eugregarinorida). Polar Biol 33: 1339-1350

Rueckert S, Leander BS (2008) Morphology and phylogeny of two novel marine gregarines (Apicomplexa, Eugregarinorida)-Lankesteria chelyosomae sp. n. and Lankesteria cystodytae sp. n. - from the intestines of North-eastern Pacific ascidians. Zool Scr 37:637-645

> Rueckert S, Leander BS (2009) Molecular phylogeny and surface morphology of marine archigregarines (Apicomplexa), Selenidium spp., Filipodium phascolosomae n. sp., and Platyproteum n. g. and comb. from North-Eastern Pacific peanut worms (Sipuncula). J Eukaryot Microbiol 56:428-439

Sasakura Y (2007) Germline transgenesis and insertional mutagenesis in the ascidian Ciona intestinalis. Dev Dyn 236:1758-1767

Sasakura Y, Awazu S, Chiba S, Satoh N (2003) Germ-line transgenesis of the Tc1/mariner superfamily transposon Minos in Ciona intestinalis. Proc Natl Acad Sci USA 100: 7726-7730

Sasakura Y, Nakashima K, Awazu S, Matsuoka T, Nakayama A, Azuma J, Satoh N (2005) Transposonmediated insertional mutagenesis revealed the functions of animal cellulose synthase in the ascidian Ciona intestinalis. Proc Natl Acad Sci USA 102: 15134-15139

Satoh N (2003) The ascidian tadpole larva: comparative molecular development and genomics. Nat Rev Genet 4: 285-295

Siedlecki M (1901) Contribution à l'étude des changements cellulaires provoqués par les gregarines. Arch Anat Microsc 4:87-100

Siva-Jothy MT, Plaistow SJ (1999) A fitness cost of eugregarine parasitism in a damselfly. Ecol Entomol 24: 465-470

Vávra J, Small EB (1969) Scanning electron microscopy of gregarines (Protozoa, Sporozoa) and its contribution to the theory of gregarine movement. J Protozool 16: 745-757

Zuk M (1987) The effects of Gregarine parasites, body size, and time of day on spermatophore production and sexual selection in field crickets. Behav Ecol 21:65-72

Submitted: July 2, 2012; Accepted: September 11, 2012 Proofs received from author(s): November 7, 2012 